\title{
La empatía y su relación con el enojo en estudiantes de medicina de la UAEH
}

\author{
Empathy and its relation to anger in medicine students from the UAEH \\ David Jiménez-Rodríguez ${ }^{\mathrm{a}}$, Fernando I. Sánchez-Flores ${ }^{\mathrm{b}}$, \\ Gabriel Eduardo Lara-Abad ${ }^{c}$,
}

\begin{abstract}
:
Currently, health professionals face a complex environment, where Human Rights and the complaint of non-conformity to an inadequate service are presented more regularly in Mexican society. For this reason, it is necessary to identify the level of empathy linking emotional regulation, specifically with the emotion of anger, in professionals in this sector, since these variables are essential in medical practice. The present study had the participation of students of the UAEH degree in medicine $(n=160)$ of the 4th, 5th and 6th semesters since from these semesters the students have interaction through their practices with patients and it is it is essential to know the level of development of these concepts in students; The instruments used for data collection were the Jefferson Medical Empathy Scale, which is based on a 7-point Likert scale, and the 10-item Anger-Trait Scale on the propensity to get upset. As main findings, it was found that there is a low relationship between anger regulation and medical empathy in UAEH medical students who carry out practices with patients.
\end{abstract}

Keywords:

Empathy, Anger, Medical Students, UAEH

Resumen:

En la actualidad los profesionales de la salud se enfrentan a un entorno complejo, donde los Derechos Humanos y la denuncia por inconformidad ante un servicio inadecuado se presentan con mayor regularidad en la sociedad Mexicana. Por este motivo es necesario identificar el nivel de empatía vinculando a la regulación emocional, específicamente con la emoción del enojo, en los profesionales de este sector, ya que estas variables son esenciales en el quehacer médico. El presente estudio tuvo una participación de estudiantes de la licenciatura de medicina de la UAEH ( $n=160)$ de los semestres $4^{\circ}, 5^{\circ}$ y $6^{\circ}$ ya que a partir de estos semestres los alumnos tienen interacción mediante sus prácticas con pacientes y es esencial conocer el nivel de desarrollo de estos conceptos en los estudiantes; los instrumentos utilizados para la recolección de los datos fueron el Escala Médica de Empatía de Jefferson que está basado en una escala Likert de 7 puntos y el Inventario Multicultural de la Expresión de la Cólera y la Hostilidad (ML-STAXI). Como principales hallazgos se encontró que existe una baja relación entre la regulación del enojo y la empatía médica en estudiantes de medicina de la UAEH que realizan prácticas con pacientes.

Palabras Clave:

Empatía, Enojo, Estudiantes de Medicina, UAEH

\section{Introducción}

La empatía es relevante para la mejora en la calidad de las interacciones relaciones personales en los distintos contextos de la vida del ser humano 1. La empatía se define simplemente como la capacidad para responder de forma afectiva o cognitivamente ante las emociones de otra persona 2.

Por su parte, la empatía médica es considerada como uno de los elementos más importantes en la relación del profesional de salud, la familia y el paciente para generar atención de calidad o una adecuada adherencia al tratamiento 3,4. Por tanto, de no crearse esta relación empática médico-paciente podría generarse insatisfacción por parte del usuario y el seguimiento del tratamiento se vería afectado 5 .

Por otra parte, Faye-Dumanget, Abdel-Halim y Marjolet 6, reportan que la falta de regulación emocional en los profesionales de la salud se relaciona directamente con la calidad de atención a los usuarios. También Sutton 7 menciona que la capacitación en el desarrollo de

\footnotetext{
a Autor de Correspondencia, Universidad Autónoma del Estado de Hidalgo, https://orcid.org/0000-0002-2686-2384, Email: david_jimenez6404@uaeh.edu.mx

b Universidad Autónoma del Estado de Hidalgo, https://orcid.org/0000-0002-9051-4907, Email: Fernando_sanchez11119@uaeh.edu.mx

cUniversidad Autónoma del Estado de Hidalgo, https://orcid.org/0000-0002-7670-7825 Email: gabriel_lara9718@uaeh.edu.mx
} 
habilidades en la regulación emocional, específicamente en la regulación y expresión del enojo, es fundamental para mejorar el involucramiento emocional y la empatía en la relación médico-paciente.

Actualmente la práctica de la medicina es la actividad profesional con mayor exigencia en normatividad y reglamentación jurídica, además como señala Zurita 8, el médico ha contribuido al nacimiento de los problemas médicos legales con su actitud desinteresada para el paciente.

Por otra parte, Guzmán 9 postula el origen de la "medicina defensiva", la cual se define como una alteración en la forma de práctica médica, inducida por amenaza o posibilidad de demanda, al momento de que el médico es demandado queda una alteración del ejercicio médico debido a la imposibilidad de retirarse del ejercicio profesional, se comienza a ejercer una medicina defensiva, se nota crueldad en la información, el rechazo a casos difíciles y valoración de familiares y pacientes como los peores enemigos del médico.

En la actualidad, los profesionales de la salud se enfrentan a un entorno complejo en el que convergen avances científicos y tecnológicos, cambios sociodemográficos y epidemiológicos, donde la cultura de los Derechos Humanos y la denuncia por la inconformidad ante un mal servicio recibido o la mala calidad de un producto, se está posicionando en la sociedad 10.

Ante este complejo escenario resulta inevitable que las prestaciones de los servicios de salud generen insatisfacciones, inconformidades, conflictos, quejas y demandas civiles 0 denuncias penales. Las consecuencias nocivas de una demanda afectan tanto al paciente como al médico. En el caso del paciente, la demanda perjudica a su familia y a su entorno laboral, social, económico y moral; sin embargo, el más perjudicado es el médico, pues más allá de enfrentar al sistema judicial, una consecuencia muy significativa es el "linchamiento social". Al médico se le considera culpable antes de que el juicio haya terminado en una sentencia. Así los efectos sociales, profesionales, psicológicos, financieros y morales del personal de salud durante y después del proceso judicial pueden llegar a ser devastadores 11.

Por esta razón el propósito central del presente estudio es conocer la relación existente entre la regulación del enojo con la empatía médica en estudiantes de medicina de la UAEH que realizan prácticas con pacientes.

Por lo anterior los resultados de la presente investigación podrán aportar nuevas expectativas orientadas a una mejora en el servicio médico 11. También es importante destacar que no existen investigaciones que correlacionen dichas variables por lo que servirá como antecedente para investigaciones futuras.

\section{Método}

Participaron 160 estudiantes de la UAEH de los semestres $4^{\circ}, 5^{\circ}$ y $6^{\circ}$.

Muestreo: Intencional por conveniencia

Diseño: No-experimental
Instrumentos: Escala Médica de Empatía de Jefferson (EEMJ) validada por Alcorta- Garza, González, Tavitas, Rodríguez y Hojat 12, validada para versión mexicana, incluye 20 ítems sobre una escala Likert de 7 puntos. Los puntajes de la EEMJ pueden fluctuar entre un mínimo de 20 a un máximo de 140; mientras más alto sea el puntaje, más empática la orientación del individuo.

El segundo instrumentos es el Inventario Multicultural de la Expresión de la Cólera y la Hostilidad (ML-STAXI) 13,14, el cual está dividido en nueve factores que se describirán a continuación: Factor 1: Control externo del enojo; Factor 2: deseo de expresar el enojo físico como verbalmente; Factor 3: Control interno del enojo como los intentos por calmarse a través de relajación; Factor 4: Reacción de enojo; Factor 5: Temperamento; Factor 6: Sentimiento de enojo; Factor 7: Enojo externo. Que puede describirse como el enojo que es expresado hacia los demás; Factores 8 y 9: abarcan control interno.

\section{Análisis de los datos}

La información se analizó siguiendo la estructura de cada instrumento, en el caso de la Escala Médica de Empatía de Jefferson se optó por realizar una sumatoria de las puntuaciones obtenidas de cada participante y de esta forma obtener una sumatoria total. Para realizar el análisis del Inventario Multicultural de la Expresión de la Cólera y la Hostilidad (ML-STAXI) se dividió al instrumento en los factores que lo componen: Control externo, Deseo, Control interno, Reacción, Temperamento, Sentimiento de enojo, Enojo Externo y Enojo Interno.

Se realizó el análisis con el Paquete Estadístico para las Ciencias Sociales (SPSS) en su versión 21 , llevando a cabo una correlación bivariada entre la sumatoria de la Escala Médica de Empatía de Jefferson y cada uno de los factores que componen el Inventario Multicultural de la Expresión de la Cólera y la Hostilidad (ML-STAXI).

\section{Resultados}

En las Tablas 1 y 2 respectivamente se muestran los resultados de los análisis estadísticos. Como se muestra en las Tablas 1 y 2 se obtuvieron bajas correlaciones con el Factor 1 que corresponde a Control Externo y en el Factor 3 que corresponde a Control Interno, por otro lado se encontró una correlación negativa en el Factor 5 que corresponde al indicador de Temperamento. Lo cual se puede entender de la siguiente manera, a mayor temperamento menor presencia de empatía médica.

Por otro lado, los rangos obtenidos en la prueba de empatía tienen como mínimo 64 y máximo 118, no se obtuvieron diferencias estadísticamente significativas entre sexo ni en los semestres en los que estudian los alumnos, dichas puntuaciones se mantuvieron constantes entre el paso de un semestre a otro.

Es decir, la ausencia de empatía se mantiene constante a lo largo de los semestres y es indistinto si el estudiante es hombre o mujer. 
Tabla 1. Correlaciones significativas

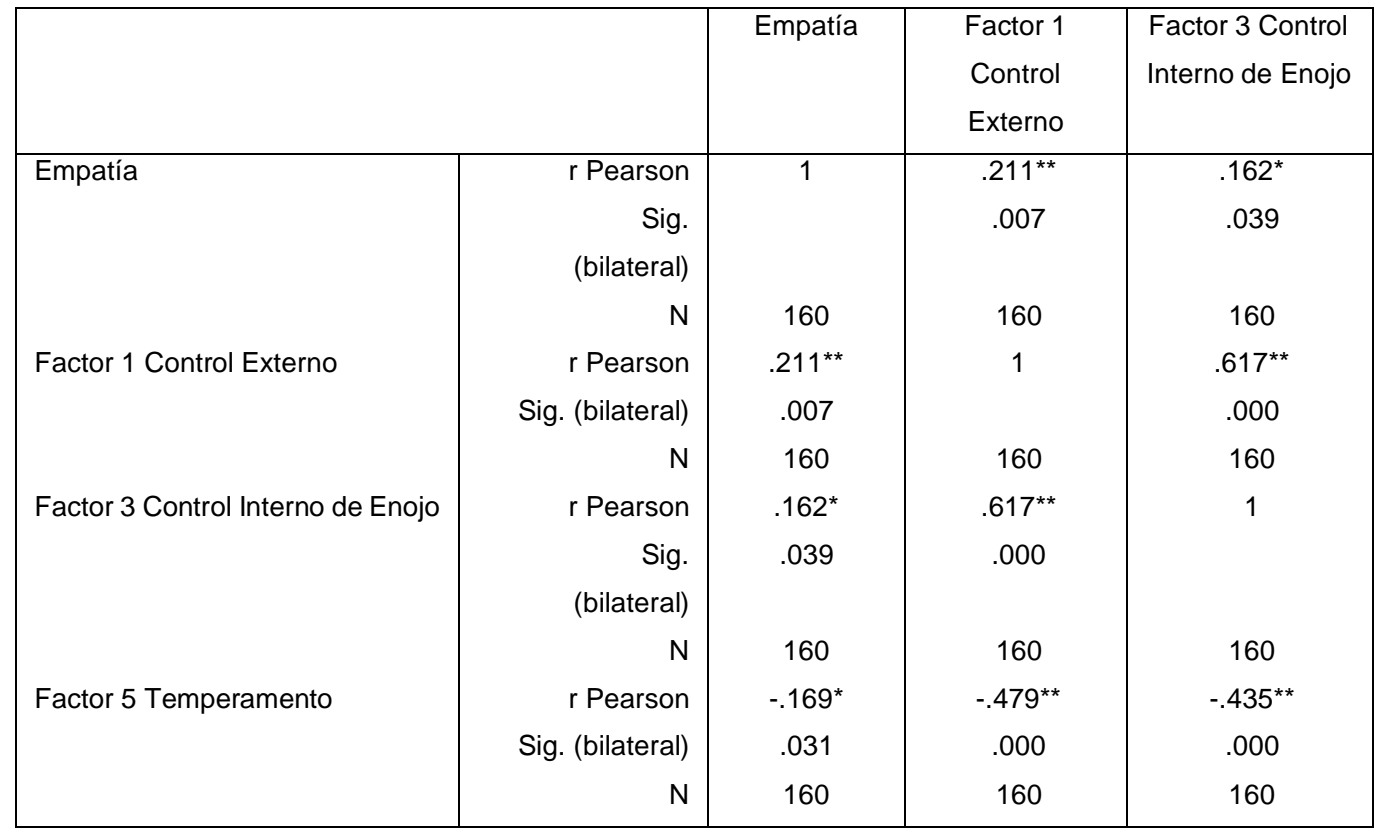

* La correlación es significante al nivel 0,05 (bilateral)

${ }^{* *}$ La correlación es significativa al nivel 0,01 (bilateral)

Tabla 2. Correlaciones significativas

\begin{tabular}{|l|c|c|}
\hline \multicolumn{2}{|c|}{} & Factor 5 Temperamento \\
\hline Empatía & Correlación de Pearson & -.169 \\
& Sig. (bilateral) & .031 \\
& $\mathrm{~N}$ & 160 \\
& Correlación de Pearson & $-.479^{\star *}$ \\
Factor 1 Control Externo 3 Control Interno de Enojo & Sig. (bilateral) & .000 \\
& $\mathrm{~N}$ & 160 \\
& Correlación de Pearson & $-.435^{*}$ \\
& Sig. (bilateral) & .000 \\
& $\mathrm{~N}$ & 160 \\
\hline
\end{tabular}

* La correlación es significante al nivel 0,05 (bilateral)

** La correlación es significativa al nivel 0,01 (bilateral)

\section{Conclusiones}

En correspondencia con el propósito de la presente investigación, podemos mencionar que existe una baja relación entre la regulación del enojo con la empatía médica en estudiantes de medicina de la UAEH que realizan prácticas con pacientes.

Los resultados obtenidos coinciden con lo que nos señala la literatura $1,2,4,5,6$, sobre que existe una relación entre la regulación de las emociones (inteligencia emocional) y la empatía. En esta etapa de constantes revisiones curriculares, existe la premisa de dotar a los futuros profesionistas de elementos que le permitan tener una adecuada comunicación con los participantes de su ejercicio profesional. Lo anterior no es exclusivo de la enseñanza médica, ni de las ciencias de la salud, sino de todas las actividades profesionales. La empatía es una habilidad social avanzada, que incluye un repertorio de conductas enfocadas a ponerse en el lugar del otro, entender la perspectiva del que está enfrente y comprender la situación general y específica por lo que atraviesa el otro individuo. La intra e interdisciplinariedad permite que los educando en formación se informen de distintas estrategias para realizar su ejercicio profesional con responsabilidad social y apegada a los estándares nacionales e internacionales de una buena praxis. 
Esta investigación contribuye a futuros estudios relacionados en el campo de la empatía médica, o bien al estudio profundo de la expresión del enojo para mejorar las interacciones que se dan entre el médico y paciente.

Los autores consideramos que existen áreas de oportunidad donde el quehacer psicológico tiene un papel primordial en la enseñanza del manejo de las emociones denominadas tanto como positivas y negativas. Se considera también que el trabajo conjunto bien organizado, trae por consecuencia un impacto positivo en los educandos en medicina.

\section{Referencias}

[1] Hernández, I., \& Dickinson, M. E. Importancia de la inteligencia emocional en Medicina. Investigación en Medicina 2014.

[2] Cadena, O., Montoya, C., Ortiz, C. La empatía y su enseñanza-aprendizaje como componente fundamental de la práctica médica. Revista de especialidades médico-quirúrgicas, 2012; 31(3): 5-8

[3] Veloz, S., Jiménez-Rodríguez, D. Programa de intervención para mejorar la adherencia al tratamiento en pacientes con enfermedad crónica. Congreso Internacional Academia Journals. Octubre 2020

[4] Márquez, A. Empatía y satisfacción de la Relación médicopaciente, 2014

[5] Cuevas L.M, Ávila I., Oliver J., Sámano M., Palomares G., Garduño J. (2013). Relación entre médico y paciente en la consulta externa de unidades de primer nivel de atención médica 2013; 33(6) 1-10

[6] Faye-Dumanget, C., Abdel-Halim, B., Marjolet, M. Efecto del burnout y del stress post-traumatizante en la empatía en el caso de los estudiantes educadores y enfermeros. Revista Electrónica Medicina, Salud y Sociedad 2015; 5(2): 83-93.

[7] Sutton, A. H., Grijalva, M. G., Maya, A. P., Barrios, P. D., Ramírez, E. S., Tellez, S. E. G., Torres, I. H. Las tres dimensiones de la comunicación médico-paciente: biomédica, emocional e identidad cultural. Revista CONAMED $2015 ; 20(1)$

[8] Zurita, G. Estado actual de las demandas. Cirujano general 2011; 33(2): 141-146.

[9] Guzmán, F. Derecho Médico: El impacto Psicológico de una Demanda Sobre el Médico. Heraldo Médico 2014; 23: 228238

[10] Robledo, H., Aguilar, T., Fajardo, G., Meljem, J. La queja médica; tema de análisis y reflexión actual. CONAMED 2012 . 17(4): 11-20.

[11] Martínez A., Chávez S., Ortega E. K. Síndrome posdemanda médico-legal. CONAMED 2016, 21(1): 55-66.

[12] Alcorta- Garza, A., González, J., Tavitas, S., Rodríguez, F, Hojat, M. Validación de la Escala de Empatía Médica de Jefferson en Estudiantes de Medicina Mexicanos. Salud Mental 2005, 28(5), 57-63

[13] Spielberger, C. D., \& Reheiser, E. C. Assessment of emotions: Anxiety, anger, depression, and curiosity. Applied Psychology: Health and Well-Being 2009; 1: 271-302.
[14] Alcázar-Olán, R. J., Deffenbacher, J. L., Byrne, Z. Assessing the Factor Structure of the Anger Expression Inventory (ML-STAXI) in a Mexican Sample 2012; 11( 2): 307318 . 